\title{
LUM Gene
}

National Cancer Institute

\section{Source}

National Cancer Institute. LUM Gene. NCI Thesaurus. Code C118986.

This gene is involved in collagen binding. 